\title{
How Reaction Time, Update Time, and Adaptation Time Influence the Stability of Traffic Flow
}

\author{
Arne Kesting \& Martin Treiber* \\ Technische Universität Dresden, Andreas-Schubert-Straße 23, 01062 Dresden, Germany
}

\begin{abstract}
When modeling the acceleration and deceleration of drivers, there are three characteristic time constants that influence the dynamics and stability of traffic flow: The reaction time of the drivers, the velocity adaptation time needed to accelerate to a new desired velocity, and the numerical update time. By means of numerical simulations with a time-continuous car-following model, we investigate how these times interplay with each other and effectively influence the longitudinal instability mechanisms for a platoon of vehicles. The long-wavelength string instability is mainly driven by the velocity adaptation time while short-wavelength local instabilities arise for sufficiently high reaction and update times. Furthermore, we investigate the relation between large update time steps and finite reaction times as they both introduce delays in the reaction to the traffic situation. Remarkably, the numerical update time is dynamically equivalent to about half the reaction time, which clarifies the meaning of the time step in models formulated as iterated maps such as the Newell and the Gipps model. Furthermore, with respect to stability, there is an optimal adaptation time as a function of the reaction time.
\end{abstract}

\section{INTRODUCTION}

Traffic dynamics, including the nature of human driving behavior and their representations in terms of mathematical models, attracts extensive interest across various scientific disciplines such as traffic engineering, physics, mathematics, and psychology (Brackstone and McDonald, 1999; Chowdhury et al., 2000; Green, 2000; Helbing, 2001; Kerner, 2004). An essential feature of human driving is a considerable reaction time, which is a consequence of the physiological aspects of sensing,

\footnotetext{
*To whom correspondence should be addressed. E-mail: treiber@
} vwi.tu-dresden.de. perceiving, deciding, and performing an action (Shiffrin and Schneider, 1977). This complex reaction time is of the order of $1 \mathrm{~s}$ and varies strongly between different drivers (age, gender), different stimuli, and different studies (Green, 2000). Remarkably, adaptive cruise control (ACC) systems, which control the acceleration (and deceleration) of a vehicle, typically display time delays on the control path that cannot be neglected either (Marsden et al., 2001; Kranke et al., 2006). Because ACC systems are the first driver assistance systems with the potential to influence traffic flow characteristics (Marsden et al., 2001; VanderWert et al., 2002; Kesting et al., 2007a,b), a profound understanding of the dynamics and the instability mechanisms caused by time delay is relevant from the point of view of automated driving as well.

Clearly, reaction times are an essential factor contributing to traffic instabilities and, consequently, are an essential element in many traffic models (May, 1990). In the most straightforward case, they are introduced as time delays into time-continuous car-following models which result in a coupled set of delay-differential equations. This approach has been pursued, for example, for the Optimal Velocity Model (Bando et al., 1995, 1998), and for the Intelligent Driver Model (Treiber et al., 2000, 2006a). More commonly, however, microscopic traffic models have been formulated in terms of an iterated coupled map such as the model of Gipps (1981), or the model of Newell (1961), or as a cellular automaton such as the model of Nagel and Schreckenberg (1992). In this class of models, the update time is considered as an explicit model parameter rather than an auxiliary parameter needed for numerical integration. Moreover, it is often interpreted as "reaction time" as well.

Even for zero reaction time and negligible update time, it is well known in the traffic theory for carfollowing models (Treiber et al., 1999), and also for 
macroscopic models (Treiber et al., 1999), that collective instabilities of the traffic flow can occur. This is true although a pair of vehicles is always locally stable in this case, cf. Equations (6)-(7) below. The reason is that, in an extended multi-particle system with many degrees of freedom, two concepts of linear stability have to be considered: Local stability is related to the response of a vehicle following the motion of the vehicle directly in front, that is, to the dynamics of a pair of vehicles. Asymptotic, string or collective stability refers to the damping of a perturbation initially introduced by the leading vehicle that propagates upstream relative to the vehicle motion in a platoon of several vehicles following each other (May, 1990) which is, in general, a more restrictive criterion than local stability. In traffic flow, the source of the string instabilities is the finite velocity adaptation time resulting from limited acceleration capabilities. As a consequence, perturbations amplify while propagating upstream in the platoon of vehicles and eventually lead to oscillating congested traffic (stop-and-go traffic), which is a commonly observed type of traffic congestion (Schönhof and Helbing, 2007).

In this article, we carefully distinguish between reaction time, update time, and adaptation time and investigate the role of each of these times with respect to instabilities of traffic flow. We identify the local and collective mechanisms for instability and show that reaction time and update time are mainly responsible for the first and the adaptation time for the latter.

The article is structured as follows. In the following section, the update time (in 2.1), the reaction time (2.2), and the velocity adaptation time (2.3) will be introduced in a general form applicable to the class of time-continuous car-following models. For matters of illustration, we will apply these concepts to the Intelligent Driver Model (Treiber et al., 2000) and the dynamic effects resulting from the three different times will be discussed (2.4). In Section 3, the stability of a platoon of vehicles will be investigated by numerical simulations. First, we will study how the stability is influenced by the reaction time and a finite acceleration capability (3.2). Second, we will investigate the interplay between reaction time and numerical update time (3.3). Finally, our results will be discussed in Section 4 .

\section{IMPLEMENTATION AND MODELING REACTION TIME, UPDATE TIME, AND ADAPTATION TIME IN CAR-FOLLOWING MODELS}

Microscopic traffic flow models describe the motion of individual driver-vehicle units $\alpha$. In this work, we will focus on the subclass of (single-lane) time-continuous microscopic models, that is, car-following models, where the acceleration $\mathrm{d} v_{\alpha} / \mathrm{d} t$ of vehicle $\alpha$ is of the general form

$$
\frac{\mathrm{d} v_{\alpha}}{\mathrm{d} t}=f\left(s_{\alpha}, v_{\alpha}, \Delta v_{\alpha}\right)
$$

The acceleration therefore depends on the own velocity $v_{\alpha}$, the gap $s_{\alpha}$, and the velocity difference (approaching rate) $\Delta v_{\alpha}:=v_{\alpha}-v_{\alpha-1}$ to the leading vehicle $\alpha-1$. Here and in the following, we assume that the vehicle indices $\alpha$ are ordered such that $\alpha-1$ denotes the preceding vehicle. The gap $s$ is defined by the vehicle positions $x$ and vehicle lengths $l$ by $s_{\alpha}(t):=x_{\alpha-1}(t)-x_{\alpha}(t)-l_{\alpha-1}$. Notice that in the model class defined by Equation (1), the acceleration only depends on the immediate predecessor and it is instantaneous in time. The specific model is uniquely defined by the acceleration function $f(\cdot)$. It is straightforward to generalize the model to include a look-ahead to several vehicles (Treiber et al., 2006a), include the vehicle immediately behind the drivers, or include reactions to decelerations of the front vehicle (braking lights). All this leads to greater stability but does not change the main results. So, for the purpose of clarity, we only consider models of the type (1). In the following subsections, we will describe how the update time, the reaction time, and the velocity adaptation time are related to models of type (1).

\subsection{Update time}

Together with the general equation of motion, $\mathrm{d} x_{\alpha} / \mathrm{d} t=$ $v_{\alpha}$, Equation (1) represents a locally coupled system of ordinary differential equations (ODEs) for the positions $x_{\alpha}$ and velocities $v_{\alpha}$ of all vehicles $\alpha$. As the considered acceleration functions $f(\cdot)$ are nonlinear, we have to solve the set of ODEs by means of numerical integration. In the context of car-following models, it is natural to use an explicit scheme assuming constant accelerations within each update time interval $\Delta t$. This leads to the update rules

$$
\begin{aligned}
& v_{\alpha}(t+\Delta t)=v_{\alpha}(t)+\dot{v}_{\alpha}(t) \Delta t: \\
& x_{\alpha}(t+\Delta t)=x_{\alpha}(t)+v_{\alpha}(t) \Delta t+\frac{1}{2} \dot{v}_{\alpha}(t)(\Delta t)^{2}
\end{aligned}
$$

where $\dot{v}_{\alpha}(t)$ is an abbreviation for the acceleration function $f\left(s_{\alpha}(t), v_{\alpha}(t), \Delta v_{\alpha}(t)\right)$. For $\Delta t \rightarrow 0$, this scheme locally converges to the exact solution of (1) with consistency orders 1 and 2 for the velocities (Euler update), and positions (modified Euler update), respectively.

Besides the numerical necessity for a finite time discretization, the update time $\Delta t$ can be interpreted as representing finite attention to the traffic: Only at times that are a multiple of $\Delta t$, drivers look at the traffic situation and instantaneously adapt their acceleration to the new situation. Because of the intuitive meaning of this update 
procedure in the context of traffic, the update rules (2), or similar rules, are sometimes considered as part of the model itself rather than as a numerical approximation. Popular examples of such coupled map models include the model of Newell (1961), and the model of Gipps (1981).

Finally, it is instructive to eliminate $\dot{v}_{\alpha}$ from the positional update of (2) by using the velocity update rule. The resulting positional update $x_{\alpha}(t+\Delta t)=x_{\alpha}(t)+\left(v_{\alpha}(t)+\right.$ $\left.v_{\alpha}(t+\Delta t)\right) \Delta t / 2$, referred to as the "trapezoidal rule," is commonly applied to close the velocity equation of the Gipps model (Wilson, 2001).

\subsection{Reaction time}

A reaction time $T^{\prime}$ is incorporated in a time-continuous model of the type given by Equation (1) by evaluating the input on the right-hand side at a previous time $t-T^{\prime}$. In this way, one obtains a coupled set of delaydifferential equations (DDEs). Although both the delay $T^{\prime}$ of time-continuous models, and the update time $\Delta t$ of iterated maps (or numerical integration schemes) have been interpreted as a reaction time, it is essential to distinguish between the two concepts. From a mathematical perspective, the analysis of DDEs is technically more demanding than that of iterated maps (Orosz et al., 2004). Moreover, the iterated map is computationally more efficient, which was the original motivation to formulate the Gipps model in this way. However, "it is not at all clear how the dynamics of the discretization are affected by the integration rule used" (Wilson, 2001) and, in particular, how it is related to the dynamics of the DDE. From a behavioral perspective, the two model classes represent different aspects of the human reaction. Although the numerical integration scheme (2) (and most iterated-map traffic models) corresponds to an instantaneous adaptation of the acceleration (or deceleration) $\dot{v}_{\alpha}$ at discrete time instances $n \Delta t$, the DDE corresponds to a delayed adaptation of the acceleration which, however, takes place continuously in time. Consequently, $\Delta t$ models the typical length of time periods where drivers are not fully concentrated on the driving task, while $T^{\prime}$ represents the actual reaction time of an ideally attentive driver.

Because the conceptual distinction between these two delay mechanisms is a main point of this contribution, we visualize the corresponding effective delay time $\tau_{\text {eff }}(t)$ as a function of continuous time $t$ (Figure 1). To compare the relative effects, both time constants have been set equal, $T^{\prime}=\Delta t=T_{0}$.

In case of the reaction time (or DDE), the acceleration at any time $t$ is calculated using the information available at $t-T^{\prime}$, that is, the delay is always given by $\tau_{\text {eff }}(t)=T^{\prime}=T_{0}$. For the complementary case of a nu-

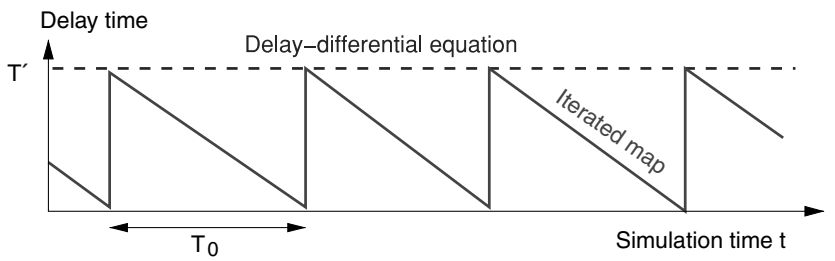

Fig. 1. Effective delay time as a function of the continuous simulation time for $T^{\prime}=T_{0}$ and $\Delta t=0$ (delay-differential equation, dashed), and for $T^{\prime}=0$ and $\Delta t=T_{0}$ (iterated map, solid).

merical update rule (2) (or iterated map) with nonnegligible update time $\Delta t=T_{0}$, the effective delay time depends on time $t$. At times $t=n \Delta t$ with integer $n$, the acceleration is instantaneously updated according to the actual positions and velocities. For all other time instants, the positional and velocity update rules (2) corresponds to constant accelerations for all vehicles, that is, to no reactions of the drivers. Consequently, any changes of the system at time $t=n \Delta t-t^{\prime}, 0 \leq t^{\prime}<\Delta t$, will be considered by the drivers at time $t=n \Delta t$ corresponding to a reaction time $\tau_{\text {eff }}\left(t^{\prime}\right)=t^{\prime}$, eventually leading to the saw-tooth function of Figure 1. Consequently, a reaction time $T^{\prime}=T_{0}$ should have a stronger destabilizing effect than an update time interval of the same numerical value. This is consistent with the results presented in Figure 8 of Section 3.3 below.

By introducing the delay $T^{\prime}$ into the numerical integration according to (2), the reaction time can be varied independently from the update interval, and the combined effects of distractions and finite reaction times can be investigated simultaneously. If the reaction time is considered as a multiple of the update time interval, that is, $T^{\prime}=n \Delta t$, it is straightforward to generalize the Equations (2) by calculating all terms on the right-hand sides with the velocities and positions $n$ time steps in the past. However, because the reaction time $T^{\prime}$ is a physiological parameter that is independent from the update time $\Delta t$ (regardless of whether the latter is interpreted physiologically or from the perspective of the numerical integration), $T^{\prime}$ is generally not a multiple of $\Delta t$. To allow for independent values of $T^{\prime}$ and $\Delta t$, we propose a linear interpolation according to

$$
x\left(t-T^{\prime}\right)=\beta x_{t-n-1}+(1-\beta) x_{t-n}
$$

where $x$ denotes any quantity on the right-hand side of Equation (1) such as $s_{\alpha}, v_{\alpha}$, or $\Delta v_{\alpha}$, and $x_{t-n}$ denotes this quantity taken $n$ time steps before the actual step. Here, $n$ is the integer part of $T^{\prime} / \Delta t$, and the weight factor of the linear interpolation is given by $\beta=T^{\prime} / \Delta t-n$. We emphasize that all input stimuli $s_{\alpha}, v_{\alpha}$, and $\Delta v_{\alpha}$ are evaluated at the delayed time, cf. Figure 3 below. As initial 
conditions, DDEs require values for the dependent variables for a whole time interval $T^{\prime}$. In the simulations, we have assumed constant initial values.

\subsection{Velocity adaptation time}

In contrast to the reaction time and the update time, the velocity adaptation time is already implicitly contained in the acceleration function of Equation (1). Because, in general, this time varies with the traffic situation, its definition is not unique. In this article, we define this time locally based on a vehicle following a leader that drives at a constant velocity $v_{\text {lead }}$. In a stationary situation, the velocity of the considered vehicle is given by $v_{\alpha}=v_{\text {lead }}$, and the distance to the leader by the equilibrium gap $s_{\alpha}=$ $s_{e}$ related to $v_{\text {lead }}$, which is calculated using the condition $f\left(s_{e}, v_{\text {lead }}, 0\right)=0$ for the acceleration function. We define the velocity adaptation time based on small deviations from stationarity for the system (1) (i.e., setting $T^{\prime}=\Delta t=0$ ) so that a local linear analysis can be applied. To perform the linearization, we split the velocity $v_{\alpha}$ into the velocity of the leading vehicle and the approaching rate $u_{\alpha}=u$, and the gap $s_{\alpha}$ into the equilibrium gap $s_{e}$, and a small deviation $y_{\alpha}=y$,

$$
\begin{aligned}
& v_{\alpha}=v_{\text {lead }}+u: \\
& s_{\alpha}=s_{e}+y
\end{aligned}
$$

From the equation $\frac{\mathrm{d} x_{\alpha}}{\mathrm{d} t}=v_{\alpha}$ defining the velocities, and Equation (1) we thus obtain

$$
\begin{aligned}
\frac{\mathrm{d} y}{\mathrm{~d} t} & =-u, \\
\frac{\mathrm{d} u}{\mathrm{~d} t} & =f\left(s_{e}+y, v_{e}+u, u\right) \\
& =\left.\frac{\partial f}{\partial s}\right|_{e} y+\left.\frac{\partial f}{\partial v}\right|_{e} u+\left.\frac{\partial f}{\partial \Delta v}\right|_{e} u+\text { nonlinear terms }
\end{aligned}
$$

The subscript $e$ denotes that all quantities are taken at the values for the stationary situation, that is, $v=v_{\text {lead }}=$ $v_{e}, s=s_{e}$, and $\Delta v=0$. The linear part can be written as a single equation for the gap deviation,

$$
\frac{\mathrm{d}^{2} y}{\mathrm{~d} t^{2}}+2 \eta \frac{\mathrm{d} y}{\mathrm{~d} t}+\omega_{0}^{2} y=0
$$

where the coefficients are given by

$$
\eta=-\left.\frac{1}{2}\left(\frac{\partial f}{\partial v}+\frac{\partial f}{\partial \Delta v}\right)\right|_{e} \text { and } \omega_{0}^{2}=\left.\left(\frac{\partial f}{\partial s}\right)\right|_{e}
$$

The second order ODE (6) is of the type for a damped linear oscillator. Using the exponential function $y(t)=$ $\exp \lambda t$ leads to the condition $\lambda^{2}+2 \eta \lambda+\omega_{0}=0$. Therefore, the velocity adaptation time can be defined by the decay time of the velocity-dominated eigenmode corresponding to the real part of the $\operatorname{root} \lambda_{2}=-\eta-\sqrt{\eta^{2}-\omega_{0}^{2}}$ according to

$$
\frac{1}{\tilde{\tau}_{v}}=\operatorname{Re}\left(-\lambda_{2}\right)=\operatorname{Re}\left(\eta+\sqrt{\eta^{2}-\omega_{0}^{2}}\right)
$$

We remark that for all meaningful definitions of acceleration functions (1) we have $\frac{\partial f}{\partial v} \leq 0$ and $\frac{\partial f}{\partial \Delta v} \leq 0$ leading to a positive value for the relaxation time (8), that is, the system is locally linearly stable, and $\tau_{v}$ is well defined. By means of simulations in Section 3.2 below, however, we will show that string instability can emerge as a result of the collective properties of traffic flow although pairs of vehicles are locally stable. Furthermore, an explicit time delay as introduced in Section 2.2 can even lead to local instability.

While $\tau_{v}$ is always nonnegative, that is, well defined, it depends on the local traffic situation as shown in Figure 2 below for a specific car-following model. To obtain a definition that is independent of the traffic density, we propose to evaluate Equation (8) for the limit of zero vehicle-vehicle interactions, that is, $s_{\alpha} \rightarrow \infty$. In this case, $\frac{\partial f}{\partial s}=0$, that is, $\omega_{0}^{2}=0$, and the limiting case $\tau_{v}$ of the
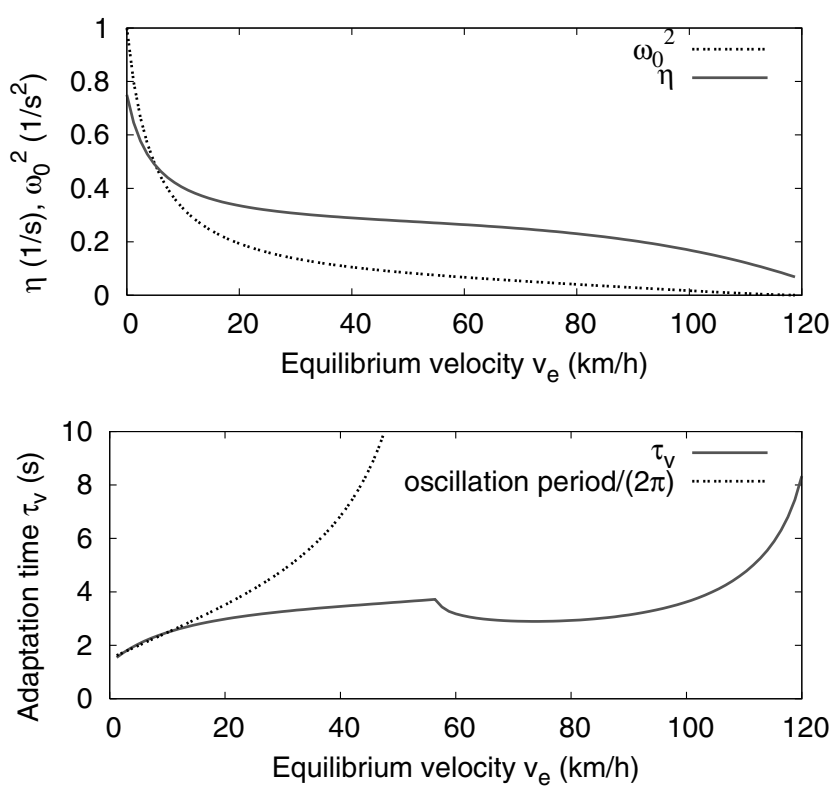

Fig. 2. Coefficients $\eta$ and $\omega_{0}^{2}$ of the damped harmonic oscillator (top), and velocity adaptation time $\tilde{\tau}_{v}$ according to Equation (8) (bottom) as a function of the equilibrium velocity $v_{e}$ for the IDM with the parameters listed in Table 1.

For $v_{e} \leq 55 \mathrm{~km} \mathrm{~h}^{-1}$, the adaptation is oscillatory and the corresponding period of the decaying oscillations is plotted as well. 
general expression $\tilde{\tau}_{v}$ for the velocity update time is given by

$$
\tau_{v}=\frac{1}{2 \eta}=\frac{-1}{\left.\left(\frac{\partial f}{\partial v}+\frac{\partial f}{\partial \Delta v}\right)\right|_{e}}
$$

For example, for the Optimal Velocity Model (Bando et al., 1995) defined by the acceleration $\dot{v}_{\alpha}=\left(v_{e}\left(s_{\alpha}\right)-\right.$ $\left.v_{\alpha}\right) / \tau$, we obtain $\tau_{v}=\tau$.

Finally, we notice that in iterated maps such as the models of Newell or Gipps providing the update of the velocity directly (and not in terms of integrating an acceleration), the velocity adaptation time is equal to the numerical update time $\Delta t$. As a consequence, the Newell model with time step $\Delta t$ is equivalent to the Optimal Velocity Model with a Euler update if the update time satisfies $\Delta t=\tau_{v}$.

\subsection{Application to the Intelligent Driver Model}

For matters of illustration, we will now apply the concept to the Intelligent Driver Model (IDM) (Treiber et al., 2000) and discuss the dynamic effects of the three times introduced in the Sections 2.1-2.3. The IDM acceleration is a continuous function incorporating different driving modes for all velocities in freeway traffic as well as city traffic. Besides the distance to the leading vehicle and the actual velocity, the IDM also takes into account velocity differences, which play an essential stabilizing role in real traffic, especially when approaching traffic jams and avoiding rear-end collisions. The IDM acceleration function is given by

$$
\begin{aligned}
\frac{\mathrm{d} v_{\alpha}}{\mathrm{d} t} & =f\left(s_{\alpha}, v_{\alpha}, \Delta v_{\alpha}\right) \\
& =a\left[1-\left(\frac{v_{\alpha}}{v_{0}}\right)^{4}-\left(\frac{s^{*}\left(v_{\alpha}, \Delta v_{\alpha}\right)}{s_{\alpha}}\right)^{2}\right]
\end{aligned}
$$

This expression combines the acceleration strategy $\dot{v}_{\text {free }}(v)=a\left[1-\left(v / v_{0}\right)^{4}\right]$ toward a desired velocity $v_{0}$ on a free road with the parameter $a$ for the maximum acceleration with a braking strategy $\dot{v}_{\text {brake }}(s, v, \Delta v)=-a\left(s^{*} / s\right)^{2}$ serving as repulsive interaction, when vehicle $\alpha$ comes too close to the vehicle ahead. If the distance to the leading vehicle, $s_{\alpha}$, is large, the interaction term $\dot{v}_{\text {brake }}$ is negligible and the IDM equation reduces to the freeroad acceleration $\dot{v}_{\text {free }}(v)$, which is a decreasing function of the velocity with the maximum value $\dot{v}(0)=a$ and the minimum value $\dot{v}\left(v_{0}\right)=0$ at the desired velocity $v_{0}$. For denser traffic, the deceleration term becomes relevant. It depends on the ratio between the effective "desired minimum gap"

$$
s^{*}(v, \Delta v)=s_{0}+v T+\frac{v \Delta v}{2 \sqrt{a b}}
$$

and the actual gap $s_{\alpha}$. The minimum distance $s_{0}$ in congested traffic is significant for low velocities only. The main contribution in stationary traffic is the term $v T$ which corresponds to following the leading vehicle with a constant desired time gap $T$. The last term is only active in nonstationary traffic corresponding to situations with $\Delta v \neq 0$ and implements an "intelligent" driving behavior including a braking strategy that, in nearly all situations, limits braking decelerations to the comfortable deceleration $b$. Note, however, that the IDM brakes stronger than $b$ if the gap becomes too small. This braking strategy makes the IDM collision-free (Treiber et al., 2000). To implement the physical limits for the braking decelerations, we restrict the maximum braking deceleration to $9 \mathrm{~ms}^{-2}$, which is a typical physical limit on dry roads. Because we have observed such values only in simulations of extreme parameter combinations leading to accidents, this limit has a negligible influence on the traffic dynamics.

All IDM parameters $v_{0}, T, s_{0}, a$, and $b$ are defined by positive values. These parameters have a reasonable interpretation, are known to be relevant, and are empirically measurable. Moreover, the parameters have realistic values, see Table 1 . The IDM has been calibrated to empirical data of several German freeways (Treiber et al., 2000). On a more microscopic level, the IDM was tested together with other microscopic models (Brockfeld et al., 2004). While all models showed large residual errors, the IDM was one of the best. Furthermore, using the same parameters as in Table 1 (apart from obvious changes for the desired velocity) both the simulated acceleration behavior from a standstill and deceleration behavior to a standstill were remarkably close to empirical observations (Wang et al., 2004, 2005).

Let us now apply the considered characteristic times to the IDM. The update time $\Delta t$ is used in the explicit integration scheme (2) and the reaction time will be

Table 1

Parameters of the Intelligent Driver Model (Treiber et al., 2000) with the values used in this paper, unless stated otherwise. The vehicle length is assumed to be $5 \mathrm{~m}$ but does not play a role. The value for the acceleration parameter $a$ is the reference; it is varied in the simulations of Section 3.2. The website http://www.traffic-simulation.de provides interactive simulations and documentation of the Intelligent Driver Model

\begin{tabular}{ll}
\hline Parameter & \multicolumn{1}{c}{ Value } \\
\hline Desired velocity $v_{0}$ & $120 \mathrm{~km} \mathrm{~h}^{-1}$ \\
Desired time gap $T$ & $1.5 \mathrm{~s}$ \\
Jam distance $s_{0}$ & $2 \mathrm{~m}$ \\
Maximum acceleration $a$ & $\mathrm{~ms}^{-2}$ \\
Desired deceleration $b$ & $\mathrm{~ms}^{-2}$ \\
\hline
\end{tabular}


(independently from $\Delta t$ ) implemented by the interpolation (3). According to Equation (9), the velocity adaptation time for the IDM is given by

$$
\tau_{v}^{\mathrm{IDM}}=\frac{v_{0}}{4 a}
$$

where $v_{0}$ is the desired velocity and $a$ the maximum acceleration parameter of the IDM. For the purpose of illustration, Figure 2 shows the actual velocity adaptation time $\tilde{\tau}_{v}$ as a function of the velocity $v_{e}$ of the leader for the parameters of Table 1 . Notice that, for most situations, $\tilde{\tau}_{v}$ is considerably lower than $\tau_{v}=8.33 \mathrm{~s}$. This is plausible because, for $v_{e}=v_{0}$, there are no constraints from other vehicles, and the relaxation is governed solely by the acceleration to the desired velocity, and not by decelerations to keep a safe distance from the leader.

The different stabilizing and destabilizing factors of the driver's behavior and the vehicle dynamics constitute a nonlinear feedback control system as visualized in Figure 3. More specifically, the controllers are the drivers, the quantities to be controlled are the velocity of the own vehicle and the distance to the leading vehicle, and the input stimuli are the observed distances and velocities, respectively (instead of the velocity difference, one can take the velocity $v_{\alpha-1}$ of the leading vehicle as equivalent input). The actions to reach desired values for the velocity and distance consist in accelerating or braking according to a car-following model, for example, Equation (10) in this article. In the framework of control theory, this acceleration is represented by a nonlinear gain function. The control path contains the times discussed in this article. When interpreting the update time $\Delta t$ to model a restricted attention to the traffic and not just as

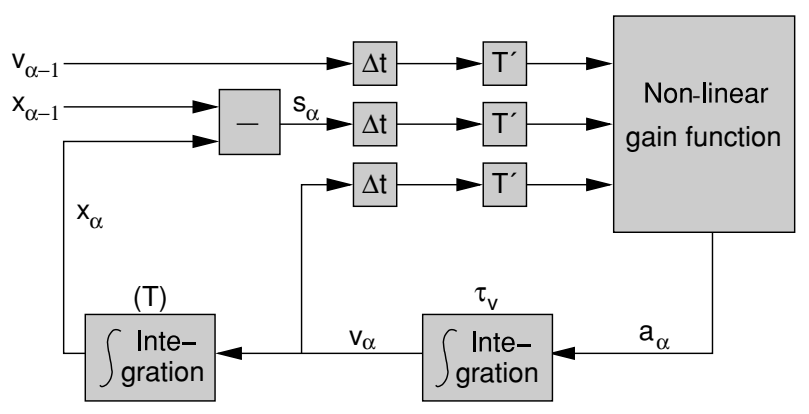

Fig. 3. Elements of the feedback loop for the vehicle dynamics of car-following models. The quantities to be controlled are the distance $s_{\alpha}$ and velocity $v_{\alpha}$. The control is performed by the acceleration function of the model representing a nonlinear gain function. The feedback path from the acceleration to the quantities to be controlled contains the characteristic times. Besides integrative elements incorporating the velocity adaptation time $\tau_{v}$, the feedback contains delay elements representing the update time $\Delta t$ and

the reaction time $T^{\prime}$ needed to calculate the acceleration function. The whole circuit is perturbed by the leading vehicle represented by the external inputs $x_{\alpha-1}$ and $v_{\alpha-1}$. a numerical parameter for integrating ODEs, its value represents the time interval where the drivers are distracted from observing the traffic. After each interval $\Delta t$, the driver looks at the traffic and takes the additional reaction time $T^{\prime}$ to come to a decision in terms of a new value for the acceleration function. Finally, it takes the additional adaptation time $\tau_{v}$ to implement this decision, that is, to approach a new velocity by means of accelerating the vehicle according to the acceleration function.

We emphasize that, in general, the parameter $T$ of the IDM describes a desired "safe" time gap and is therefore not equivalent to any of the three other times $T^{\prime}, \Delta t$, or $\tau_{v}$ although it has been set equal to each of these times in the literature (cf. the review of Helbing (2001)). While the desired time gap $T$ is a characteristic parameter of the driving style, the reaction time $T^{\prime}$ is essentially a physiological parameter and, consequently, at most weakly correlated with $T$. The time gap has, however, a strong influence on the stability of traffic flow because it determines the upper limit for the cumulative time delays of the control path from the acceleration to the desired distance.

\section{SIMULATING TRAFFIC INSTABILITY}

\subsection{Simulation setup}

We have simulated a platoon of 100 vehicles on a single lane following a leader with externally prescribed velocity $v_{\text {lead }}(t)$. As the car-following model, we have used the IDM with parameters given in Table 1. Initially, and for the first 1000s of simulation time, the leader drives constantly at $v_{\text {lead }}=25 \mathrm{~ms}^{-1}$. Furthermore, all followers are in equilibrium, that is, the initial velocities of all platoon vehicles are equal to $v_{\text {lead }}$ and the gaps equal to $s_{e}\left(v_{\text {lead }}\right)$, such that the initial model accelerations are equal to zero. To trigger possible instabilities, the externally controlled vehicle decelerates from $25 \mathrm{~ms}^{-1}$ to $19 \mathrm{~ms}^{-1}$ during the time interval $1000 \mathrm{~s} \leq t \leq 1003 \mathrm{~s}$, and drives at $v_{\text {lead }}^{\prime}=19 \mathrm{~ms}^{-1}$ afterwards. The nonlinear dynamics resulting from this finite perturbation cannot be handled by linearization. Furthermore, to our knowledge, there exists no generalized potential for applying standard methods of nonlinear stability analysis such as the theorems of Lyapunov or Krasovskii. Therefore, we will investigate the system numerically. We have also investigated more realistic scenarios with heterogeneous driver-vehicle populations by dividing the driver-vehicle units into two or more groups (e.g., "cars" and "trucks") with different values for the desired velocity $v_{0}$, the time gap $T$, the acceleration parameters $a$ and $b$, and the reaction times (the update time was always unique). We found that the global traffic dynamics and the stability behavior is essentially equivalent to using identical 
driver-vehicle units with parameters equal to the arithmetic mean of the population.

\subsection{Instability due to reaction time and finite acceleration}

We have investigated the interplay between the reaction time $T^{\prime}$ and the adaptation time $\tau_{v}$ by simulating the platoon using a constant update time of $\Delta t=0.1 \mathrm{~s}$. For this value, the numerical results represent, to a good approximation, the exact solution of the set of delay-differential equations allowing an investigation independent from the effects of the update time (which will be investigated in the following section). According to Equation (12), the velocity adaptation time for the IDM is mainly influenced by the parameter for the maximum acceleration $a$ and the desired velocity $v_{0}$. For sufficiently low values of $a$, that is, sufficiently high values for $\tau_{v}$, the resulting time delay leads to the well-known collective instabilities, even for zero reaction time (cf. Section 1). Clearly, additional delay caused by a finite reaction time $T^{\prime}$ contributes to traffic instabilities as well. Furthermore, it is expected that stability always decreases when $T^{\prime}$ increases.

We have simulated the system without reaction time $\left(T^{\prime}=0 \mathrm{~s}\right)$ and for a fixed reaction time of $T^{\prime}=0.9 \mathrm{~s}$, and three values for the acceleration parameter $a$. The results are the following:

(1) For $a=1.0 \mathrm{~ms}^{-2}$, the system is string stable, that is, the initial perturbation of $2.0 \mathrm{~m} / \mathrm{s}^{-2}$ dissipates quickly as shown in the acceleration time series for several vehicles of Figure 4 both for $T^{\prime}=0 \mathrm{~s}$ and $T^{\prime}=0.9 \mathrm{~s}$.

(2) After lowering the acceleration parameter to $a=$ $0.3 \mathrm{~ms}^{-2}$, the initial perturbation leads to a small temporary acceleration response for the immediate follower (the system is locally stable) and also for the next followers up to vehicle 10 , but the accelerations increase again for the subsequent vehicles, and finally lead to a traffic breakdown in the neighborhood of vehicle 100 at a simulated time $t \approx 1250 \mathrm{~s}$, that is, the system is string unstable as shown in Figure 5(a). After the first traffic breakdown, further stop-and-go waves develop (not shown here).

(3) Remarkably, after increasing the acceleration from the reference value $1.0 \mathrm{~ms}^{-2}$ to $a=$ $2.5 \mathrm{~ms}^{-2}$, the system becomes string unstable as well, cf. Figure 5(b). Again, further stop-andgo waves develop in the course of time further upstream. (a) String stable with settings $T^{\prime}=0.0 \mathrm{~s}, a=1 \mathrm{~m} / \mathrm{s}^{2}$

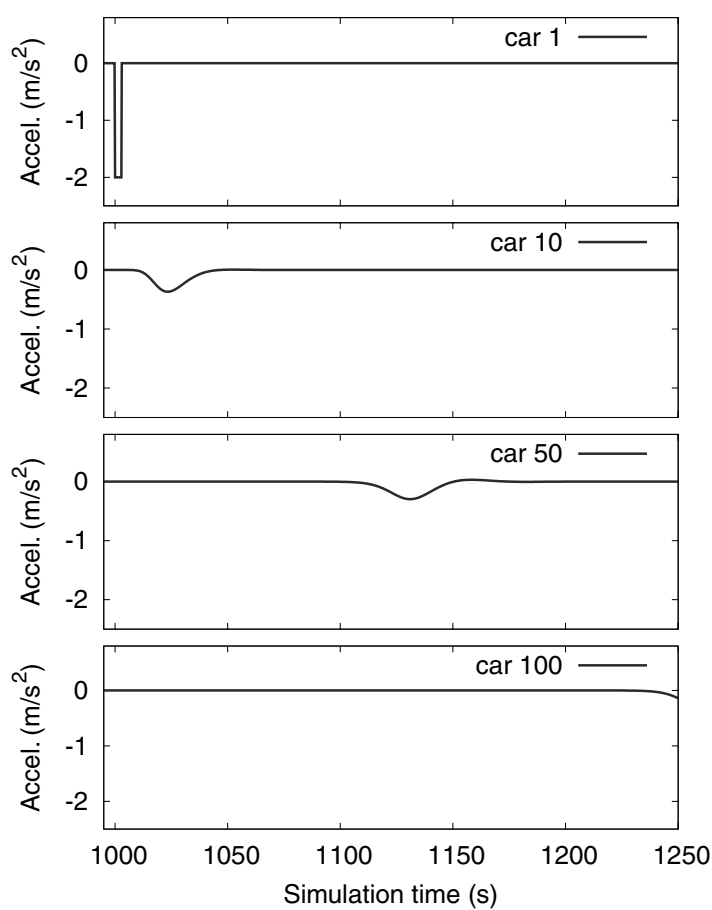

(b) String stable with settings $T^{\prime}=0.9 \mathrm{~s}, \mathrm{a}=1 \mathrm{~m} / \mathrm{s}^{2}$

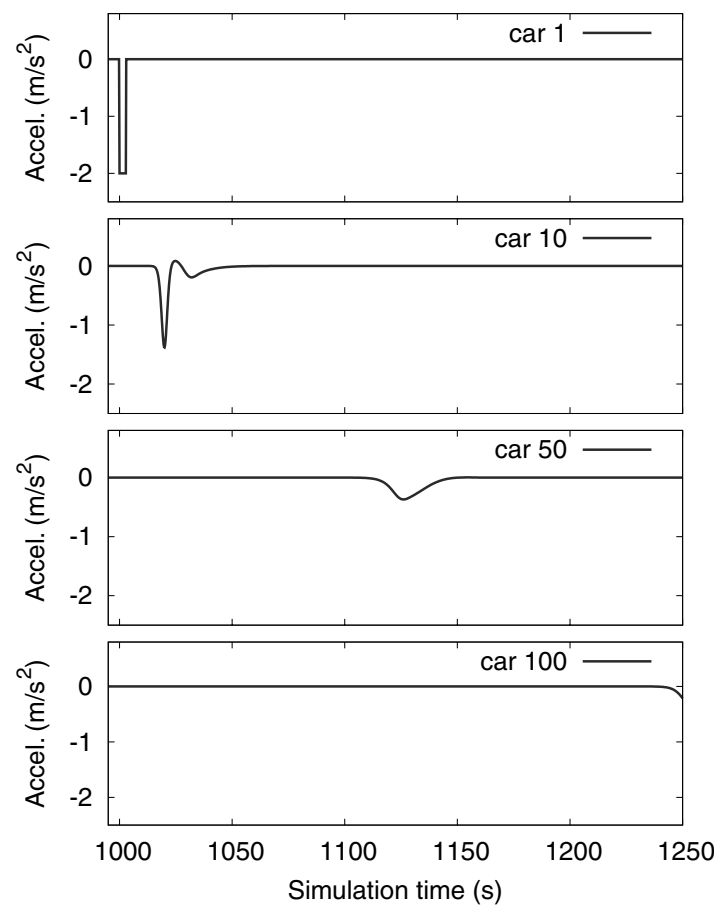

Fig. 4. Time series of the acceleration for selected platoon vehicles for zero reaction time $T^{\prime}=0 \mathrm{~s}(\mathrm{a})$, and $T^{\prime}=0.9 \mathrm{~s}(\mathrm{~b})$. The IDM acceleration parameter is set to $a=1.0 \mathrm{~ms}^{-2}$. The first vehicle induces a perturbation due to the braking maneuver at $t=1000 \mathrm{~s}$.

The initial perturbation dissipates while propagating through the platoon of vehicles, that is, the system is string stable. 
(a) String unstable with settings $\mathrm{T}^{\prime}=0.9 \mathrm{~s}, \mathrm{a}=0.3 \mathrm{~m} / \mathrm{s}^{2}$
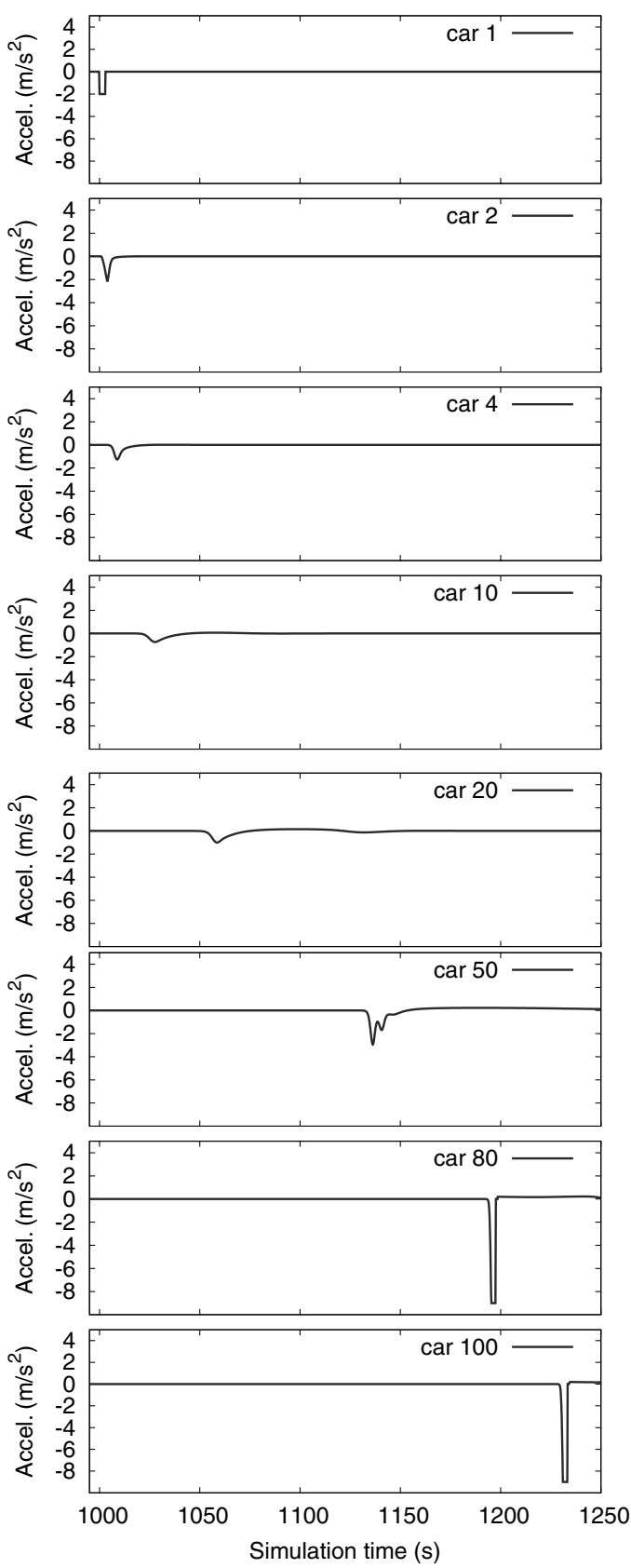

(b) String unstable with settings $\mathrm{T}^{\prime}=0.9 \mathrm{~s}, \mathrm{a}=2.5 \mathrm{~m} / \mathrm{s}^{2}$
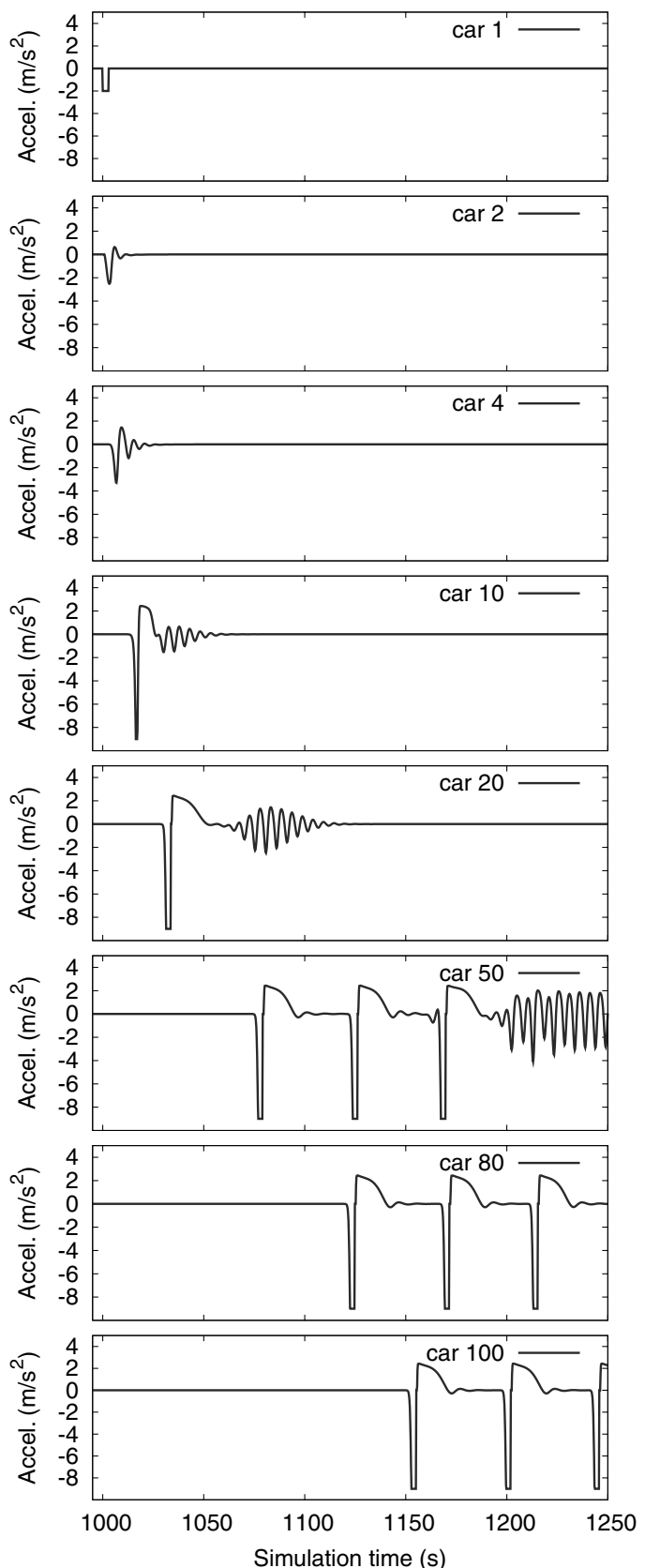

Fig. 5. Time series of the acceleration for the same scenario as in Figure 4(b) with $T^{\prime}=0.9 \mathrm{~s}$, except for the IDM parameter for the maximum acceleration being reduced to $a=0.3 \mathrm{~ms}^{-2}$ (a) or increased to $a=2.5 \mathrm{~ms}^{-2}$ (b). The system is unstable in both cases displaying long-wavelength collective instability and short-wavelength local instability for $a=0.3 \mathrm{~ms}^{-2}$ and $a=2.5 \mathrm{~ms}^{-2}$, respectively. In both scenarios, more stop-and-go waves are triggered further upstream.

When varying the maximum acceleration capability, we come to the remarkable result that stability reaches its maximum for a certain range of values for $a$ that depends on the reaction time $T^{\prime}$. Traffic flow becomes more unstable if the value of the maximum acceleration is higher or lower than this value. We checked if these results are robust with respect to parameter changes and found no qualitative difference for other parameter sets within a reasonable range. More specifically, by scaling the IDM equations (10) and (11) with the delay conditions (2) and (3) according to the Buckingham theorem, conditions for an invariant dynamic and stability 
behavior can be derived analytically. Here, it is appropriate to scale time in units of the desired time gap $T$, and space in units of $v_{0} T$. The resulting scaled equations have the same form as the original expressions when setting the scaled parameters for the desired velocity and time gap to unity, $\tilde{v}_{0}=\tilde{T}=1$, and replacing the remaining IDM parameters by the scaled quantities $\tilde{a}=T a / v_{0}, \tilde{b}=T b / v_{0}, \tilde{s}_{0}=s_{0} /\left(v_{0} T\right)$, and the delay parameters by $\tilde{T}^{\prime}=T^{\prime} / T$, and $\Delta \tilde{t}=\Delta t / T$. For example, when changing the desired time gap parameter from $T=$ $1.5 \mathrm{~s}$ to $T=0.75 \mathrm{~s}$, the stability remains unchanged if $T^{\prime}, \Delta t$, and $s_{0}$ are divided by a factor of two, while the accelerations $a$ and $b$ are multiplied by this factor.

In any case, the results are markedly different from the case of zero reaction time where higher values for $a$ (lower values for $\tau_{v}$ ) always increase the stability. This can be understood by recognizing that there are two different types of instabilities. The "classical" collective instability mechanism of the IDM is caused by the time delay due to finite accelerations that is described by the velocity adaptation time $\tau_{v}$. This instability mechanism becomes active for sufficiently low accelerations (high values of $\tau_{v}$ ) and depends only weakly on the reaction time $T^{\prime}$. As suggested by the simulation results, we will call this type of instability the long-wavelength string instability.

For finite reaction times, an additional instability mechanism becomes active for sufficiently high accelerations, that is, low values of $\tau_{v}$. Based on the simulation results, we will call this type of instability the short-wavelength instability. The two instability types are qualitatively different from each other in the following respects:

- The short-wavelength instability requires finite values for at least one of the times $T^{\prime}$ or $\Delta t$, while the longwavelength instability hardly depends on these times.

- The short-wavelength instability can appear as a local instability while the long-wavelength instability always has the nature of a string instability. Particularly, for $T^{\prime} \rightarrow 0$ and $\Delta t \rightarrow 0$ (where only the longwavelength instability is possible), the system is always locally stable, that is, the time constant defined by Equation (8) is positive.

- The short-wavelength instability is favored by an agile driving style (high accelerations) while a more "sluggish" style favors the long-wavelength instability.

Figure 5a shows the emergence of the long-wavelength instability in the plots for the cars 10, and 20. Secondary instabilities of shorter wavelengths appear only in the nonlinear regime (car 50) before a complete breakdown is observed (car 80).

In contrast, the dominating modes of the second instability mechanism have a shorter wavelength as can be seen from Figure $5 b$ for the vehicle sequence 1, 4, and 10. The range for the parameters $T^{\prime}$ and $\tau_{v}$ for the second mechanism is plausible when recognizing that the initial local instability is of the same type as that for simple feedback loops with delay-time elements, that is, the velocity adaptation time in our context. Such systems become unstable if the ratio $T^{\prime} / \tau_{v}$ exceeds a certain value of the order of unity depending on the specific model, that is, the system becomes more susceptible to this type of instability if $\tau_{v}$ decreases. In contrast, the system becomes more susceptible to the classical long-wavelength instability for increasing values of $\tau_{v}$ since the velocity adaptation time is the main source for this type of instability. Notice that these considerations are valid for a wide class of car-following models.

We have investigated this observation more systematically by calculating the instability of the system as a function of the reaction time and the acceleration parameter. To obtain a continuous measure for the instability, we have calculated the variance of the accelerations for the 20 cars $5,10,15, \ldots, 100$ based on the time series for $t>1000 \mathrm{~s}$, that is, after having applied the perturbation. Figure 6a shows this instability measure as a function

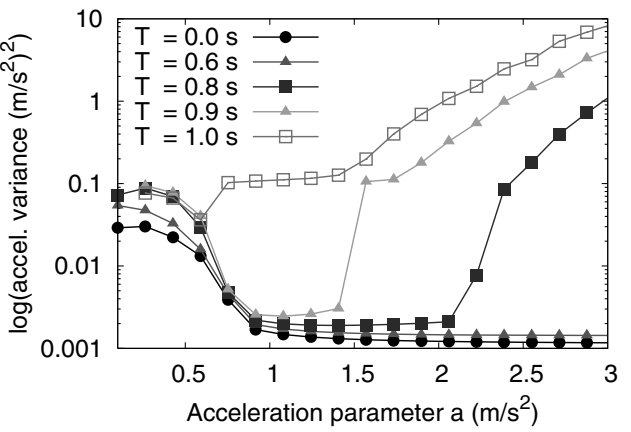

Fig. 6. System instability measured in terms of the acceleration variance (see the main text) as a function of the reaction time $T^{\prime}$ (left) and the acceleration parameter $a$ (right) for some fixed values of $a$ and $T^{\prime}$, respectively. Notice that each point represents a simulation run. 
of $T^{\prime}$ for several fixed values of $a$. We get the following results:

- For $a \geq 1 \mathrm{~ms}^{-2}$, the system is stable for sufficiently small reaction times and the instability threshold $T_{c}^{\prime}(a)$ decreases with increasing $a$, which is a signature of the short-wavelength instability mechanism.

- For $a=0.5 \mathrm{~ms}^{-2}$, the system becomes unstable regardless of the value of $T^{\prime}$, and the instability measure has only a weak dependence on $T^{\prime}$. This is a signature of the long-wavelength mechanism.

Obviously, for a given reaction time $T^{\prime}$, there is a certain optimal value for $a$, or a range of values, where the system has maximum stability. This is depicted in Figure $6 \mathrm{~b}$ where the stability is plotted as a function of $a$ for several fixed values of $T^{\prime}$ :

- For $T^{\prime} \leq 0.9 \mathrm{~s}$, the two mechanisms of instability are separated by an optimal range of the parameter $a$ where the system is completely stable. While, in the long-wavelength instability range $a \lesssim 0.6 \mathrm{~ms}^{-2}$, the instability measure depends only weakly on $T^{\prime}$, the critical acceleration $a_{c}$ at the threshold of the short-wavelength instability decreases strongly with increasing $T^{\prime}$. For $T^{\prime}<0.6 \mathrm{~s}$, this instability mechanism is no longer observed for realistic values of $a$.

- For $T^{\prime}=1.0 \mathrm{~s}$, there is no longer a range of $a$ for complete stability. Instead, both mechanisms seem to be effective simultaneously in the range of the accelerations that represented the optimal range for $T^{\prime}=0.9 \mathrm{~s}$.

These findings are summarized in the phase diagram shown in Figure 7 spanned in parameter space by the parameters $T^{\prime}$ and $a$. Because the initial perturbation leads to a finite acceleration variance for stable traffic as well, the stable phase has been identified by values for the acceleration variance below $0.003\left(\mathrm{~ms}^{-2}\right)^{2}$.

\subsection{Relation between reaction time and numerical update time}

In the simulations of Section 3.2, the update time step was so small $(\Delta t=0.1 \mathrm{~s})$ that it did not have a significant influence as confirmed by simulations with a smaller update time step of, for example, $\Delta t=0.01 \mathrm{~s}$. Investigating much larger update time steps is interesting because both finite reaction times $T^{\prime}$ and update time steps $\Delta t$ introduce delays in the reaction to the traffic situation, while, a priori, it is not clear if both effects are dynamically equivalent and, if so, for which pair of values. Moreover, such an equivalence is essential for interpreting the update time steps of coupled-map models as a reaction time. Finally, for large $\Delta t$, car-following models become numerically very efficient.

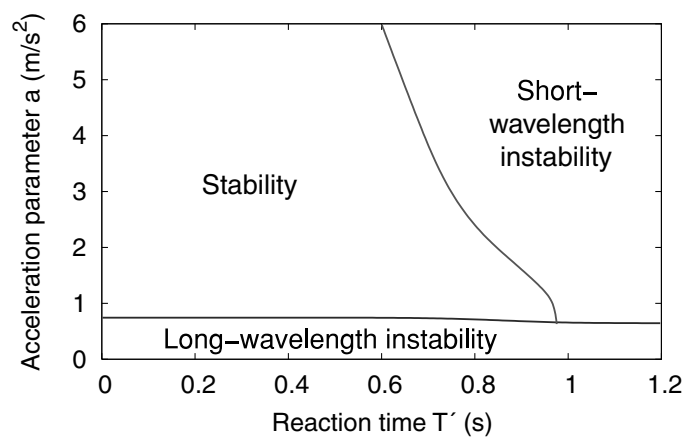

Fig. 7. Phase diagram of stable and unstable traffic flow spanned by the reaction time $T^{\prime}$ and the acceleration parameter $a$. The initial perturbation leads to the long-wavelength collective instability for small values of $a$. For higher settings of $a$, the finite reaction time causes short-wavelength local instabilities. For a broad range of combinations of $\left(T^{\prime}, a\right)$, the traffic dynamics of the vehicle platoon is stable. Interestingly, for higher reaction times, an effectively lower setting of $a$ is able to reduce the instability of the system due to delayed response to the input stimuli.

We have systematically investigated the effects of various combinations of $T^{\prime}$ and $\Delta t$ for the system presented above. Figure 8 shows the results in the form of a dynamical phase diagram spanned by both times for two settings of the IDM acceleration parameter $a$, while keeping all other parameters constant (see Table 1). The three dynamic phases are characterized by (1) no instabilities, (2) instabilities, which may be of the short-wavelength or the collective long-wavelength type but do not lead to crashes, and (3) instabilities that eventually lead to crashes.

Interestingly, for a given value $T_{0}$ of either $T^{\prime}$ or $\Delta t$, the combination $\left(T^{\prime}=T_{0}, \Delta t \approx 0\right)$ leads to a stronger destabilizing effect than the combination $\left(T^{\prime}=0, \Delta t=\right.$ $\left.T_{0}\right)$. To explain this finding, it is essential to distinguish between the reaction time $T^{\prime}$ and the update time $\Delta t$ conceptually:

(i) The limiting case $\Delta t \rightarrow 0$ for finite values $T^{\prime}=$ $T_{0}$ corresponds to the exact solution of the timecontinuous model for a finite reaction time, that is, to the delay-differential equation (3).

(ii) The case $\Delta t=T_{0}$ and $T^{\prime}=0$ corresponds to the numerical solution according to the used integration scheme for zero reaction time, that is, to a coupled iterated map.

Because the two limiting cases lead to qualitatively different mathematical models they are obviously not equivalent. Consequently, the phase boundaries of Figure 8 are not symmetric with respect to the axes. For example, the parameter combination $\left(\Delta t=1 \mathrm{~s}, T^{\prime}=0.5 \mathrm{~s}\right)$ corresponds to stable traffic while $\left(\Delta t=0.5 \mathrm{~s}, T^{\prime}=1 \mathrm{~s}\right)$ 
(a) Acceleration parameter $\mathrm{a}=1.0 \mathrm{~m} / \mathrm{s}^{2}$

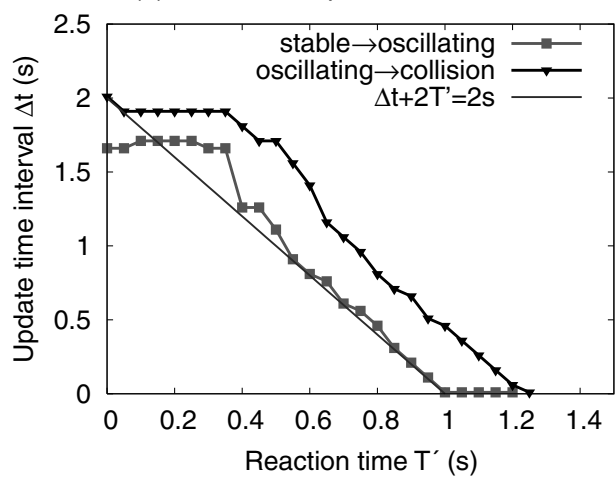

(b) Acceleration parameter $\mathrm{a}=2.5 \mathrm{~m} / \mathrm{s}^{2}$

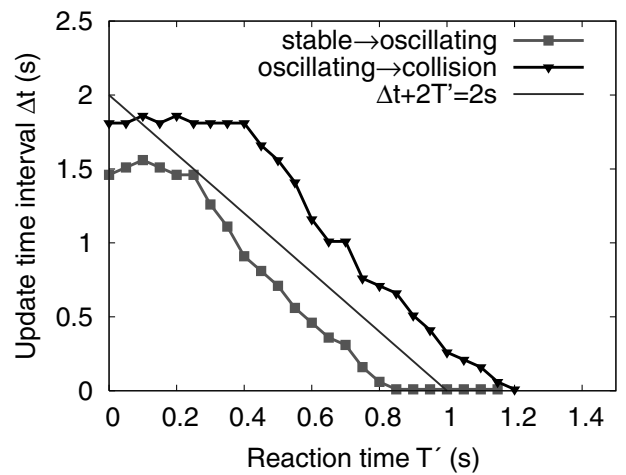

Fig. 8. Phase diagrams of the three dynamic phases for a platoon size of 100 vehicles as a function of reaction time $T^{\prime}$ and numerical time discretization $\Delta t$. Besides the numerical necessity for a finite $\Delta t$, the value of $\Delta t$ can be interpreted as "attention span," that is, as a typical length of time period where drivers do not draw their attention to the driving task.

leads to crashes. Therefore, for the same numerical values, the reaction time introduces stronger destabilizing effects than the update time.

Remarkably, the borderline between stable and oscillatory platoons is approximately given by $\Delta t+2 T^{\prime}=$ $C$ with, for example, $C=2 \mathrm{~s}$ in Figure $8 \mathrm{a}$. This means, the destabilizing effect of a finite reaction time is about twice that of a finite update time interval of the same numerical value, or, cum grano salis, the effective reaction time introduced by a finite update time interval is about $T_{\text {eff }}^{\prime} \approx \Delta t / 2$. This is consistent with the considerations summarized in Figure 1.

This interchangeability between update time and reaction time allows for an important conclusion regarding the numerical efficiency of the models: Because in contrast to simulating DDEs, large numerical update times are numerically efficient, one can simulate a given reaction time $T^{\prime}$ by iterated maps or by time-continuous models of the type (1) together with the trapezoidal numerical update scheme (2) by choosing an update time $\Delta t=2 T^{\prime}$. Modeling reaction times in this way, however, is restricted to identical reaction times for all drivers.

\section{DISCUSSION AND CONCLUSIONS}

We have investigated the three characteristic time constants that influence the dynamics and stability of traffic flow: (1) The delay caused by the finite reaction time of the drivers, (2) the time lag due to a finite velocity adaptation time needed to accelerate to a new desired velocity, and (3) the numerical update time. We presented a general microscopic modeling approach for the independent implementation of these three times for timecontinuous car-following models, that is, models that are represented by ordinary differential equations. Apart from the IDM (Treiber et al., 2000), examples include the Optimal Velocity Model (Bando et al., 1995), or the Velocity Difference Model (Jiang et al., 2001).

Introducing reaction times to this class of traffic models lead to time-delayed differential equations. On the other hand, simulating such models with zero reaction time and using an explicit integration scheme with comparatively large update time is equivalent to simulating iterated maps such as the model of Gipps (1981), or the model of Newell (1961). Often, the update time of these models is interpreted as reaction time as well, although, iterated maps are qualitatively different from delay-differential equations. Moreover, in the absence of these two times, traffic instabilities can also be caused by the finite time needed to adapt the velocity to a new desired value (as demonstrated, e.g., by the IDM), and also this time is sometimes called "reaction time."

In this article, we have clarified the role of each of these times by simulating the local and string stability of a platoon of vehicles based on the Intelligent Driver Model (IDM) for various combinations of the three times. We used the IDM just as an example. The results are valid for other time-continuous and coupled-map car-following models as well. We found that, in fact, the reaction time and the update time have a similar dynamic effect because both introduce instabilities via "short-wavelength mechanisms" that can be both local or collective in nature, while the velocity adaptation time triggers instabilities exclusively via collective long-wavelength instabilities. Moreover, we have shown that the instability effect of finite update times of numerical integration schemes is comparable to that of delay-differential equations when the value of the reaction time is half that of the update time. This is consistent with the considerations summarized in Figure 1 and the fact that the update rule (2) is equivalent to the trapezoidal rule, that is, updating the 
positions by using the average of the "old" and "new" velocities. Because this rule is applied in most iterated maps such as the Newell model, the "reaction time" $\Delta t$ of such models corresponds, in fact, only to about half the actual reaction time delay.

Furthermore, it is more plausible to interpret the value of $\Delta t$ as a typical length of time period where drivers do not draw their attention to the driving task and, consequently, do not perform actions such as adapting the acceleration to the actual situation. Therefore, finite update times $\Delta t$ can be used to model distractions and a "restricted attention span" that, in addition to reaction times, may play an important role in the driving behavior of humans (Boer, 1999).

When comparing the reaction time with the velocity adaptation time, we obtain the interesting result that the optimal acceleration (and deceleration) to obtain a maximum of stability depends on the reaction time: The higher the reaction time, the lower the optimal accelerations. Therefore, a finite reaction time of $1 \mathrm{~s}$ can be partially compensated by an optimal, that is, effectively lowered acceleration capability. This is consistent with the observation that people with comparatively long reaction times typically drive less aggressively than the average. Note, however, that, for $T^{\prime} \leq 0.8 \mathrm{~s}$, the minimum is rather flat.

With the advent of driver-assistance systems for (partly) automated driving such as autonomous accelerating and braking by means of adaptive cruise control (ACC), an understanding of the different mechanisms leading to traffic instabilities and their implementation in terms of mathematical models becomes even more relevant. It is crucial to understand the effects of ACC systems on the capacity and stability of traffic flow at an early stage so that their design can be adjusted before adverse traffic effects will be widely manifested. Both human drivers and ACC systems show finite delay times although the origins are fundamentally different. In real ACC systems, they are the result of a complex control path involving the motor control unit and the braking control system (Kranke et al., 2006). While the human reaction times generally are higher, human drivers compensate for the destabilizing effects of reaction time by anticipation, for example, by looking several vehicles ahead, anticipating the future traffic situation, and adapting to the traffic environment (Treiber et al., 2006a, 2006b). It is a current research topic to introduce at least temporal anticipation to ACC systems.

\section{REFERENCES}

Bando, M., Hasebe, K., Nakayama, A., Shibata, A. \& Sugiyama, Y. (1995), Dynamical model of traffic congestion and numerical simulation, Physical Review E, 51, 1035-42.
Bando, M., Hasebe, K., Nakanishi, K. \& Nakayama, A. (1998), Analysis of optimal velocity model with explicit delay, Physical Review E, 58, 5429.

Boer, E. (1999), Car following from the driver's perspective, Transportation Research Part F: Traffic Psychology and Behaviour, 2, 201-206.

Brackstone, M. \& McDonald, M. (1999), Car-following: a historical review, Transportation Research Part F: Traffic Psychology and Behaviour, 2, 181-96.

Brockfeld, E., Kühne, R. D. \& Wagner, P. (2004), Calibration and validation of microscopic traffic flow models, Transportation Research Record: Journal of the Transportation Research Board, 1876, 62-70.

Chowdhury, D., Santen, D. \& Schadschneider, A. (2000), Statistical physics of vehicular traffic and some related systems, Physics Reports, 329, 199-329.

Gipps, P. G. (1981), A behavioural car-following model for computer simulation, Transportation Research Part B: Methodological, 15, 105-11.

Green, M. (2000), "How long does it take to stop?" Methodological analysis of driver perception-brake times, Transportation Human Factors, 2, 195-216.

Helbing, D. (2001), Traffic and related self-driven manyparticle systems, Reviews of Modern Physics, 73, 1067-141.

Jiang, R., Wu, Q. \& Zhu, Z. (2001), Full velocity difference model for a car-following theory, Physical Review E, 64, 017101.

Kerner, B. S. (2004), The Physics of Traffic. Springer, Heidelberg.

Kesting, A., Treiber, M., Schönhof, M. \& Helbing, D., (2007a), Extending adaptive cruise control (ACC) towards adaptive driving strategies, Transportation Research Record: Journal of the Transportation Research Board, in print.

Kesting, A., Treiber, M., Schönhof, M., Kranke, F. \& Helbing, D. (2007b), Jam-avoiding adaptive cruise control (ACC) and its impact on traffic dynamics, in Schadschneider, A. Pöschel, T., Kühne, R. Schreckenberg, M. and Wolf, D. E. (eds.), Traffic and Granular Flow '05. Springer, Berlin, pp. 63343.

Kranke, F., Poppe, H., Treiber, M. \& Kesting, A. (2006), Fahrerassistenzsysteme zur aktiven Stauvermeidung im Straßenverkehr, in M. Lienkamp (ed.), VDI-Berichte zur 22. VDI/VW Gemeinschaftstagung: Integrierte Sicherheit und Fahrerassistenzsysteme. Vol. 1960. Verein Deutscher Ingenieure (VDI), Wolfsburg, p. 375.

Marsden, G., McDonald, M. \& Brackstone, M. (2001), Towards an understanding of adaptive cruise control, Transportation Research Part C: Emerging Technologies, 9, 33-51.

May, A. D. (1990), Traffic Flow Fundamentals. Prentice Hall, Eaglewood Cliffs, N.Y.

Nagel, K. \& Schreckenberg, M. (1992), A cellular automaton model for freeway traffic, Journal of Physics I France, 2 , 2221-29.

Newell, G. (1961), Nonlinear effects in the dynamics of car following, Operations Research, 9, 209.

Orosz, G., Wilson, R. E. \& Krauskopf, B. (2004), Global bifurcation investigation of an optimal velocity traffic model with driver reaction time, Physical Review E, 70(2), 26207.

Schönhof, M. \& Helbing, D. (2007), Empirical features of congested traffic states and their implications for traffic modeling, Transportation Science, 41, 1-32.

Shiffrin, R. \& Schneider, W. (1977), Controlled and automatic human information processing II: perceptual learning, automatic attending, and a general theory, Psychological Review, 84, 127-90. 
Treiber, M., Hennecke, A. \& Helbing, D. (1999), Derivation, properties, and simulation of a gas-kinetic-based, non-local traffic model, Physical Review E, 59, 239-53.

Treiber, M., Hennecke, A. \& Helbing, D. (2000), Congested traffic states in empirical observations and microscopic simulations, Physical Review E, 62, 1805-24.

Treiber, M., Kesting, A. \& Helbing, D. (2006a), Delays, inaccuracies and anticipation in microscopic traffic models, Physica $A, 360,71-88$.

Treiber, M., Kesting, A. \& Helbing, D. (2006b), Understanding widely scattered traffic flows, the capacity drop, and platoons as effects of variance-driven time gaps, Physical Review E, 74, 016123.

VanderWerf, J., Shladover, S., Miller, M. \& Kourjanskaia, N. (2002), Effects of adaptive cruise control systems on high- way traffic flow capacity, Transportation Research Record: Journal of the Transportation Research Board, 1800, 7884.

Wang, J., Dixon, K., Li, H. \& Ogle, J. (2004), Normal acceleration behaviour of passenger vehicles starting from rest at allway stop-controlled intersections, Transportation Research Record: Journal of the Transportation Research Board, 1883, 158-66.

Wang, J., Li, H. \& Ogle, J. (2005), Normal deceleration behaviour of passenger vehicles at stop sign-controlled intersections, Transportation Research Record: Journal of the Transportation Research Board, 1937, 120-27.

Wilson, R. E. (2001), An analysis of Gipps's car-following model of highway traffic, IMA Journal of Applied Mathematics, 66(5), 509. 